\title{
Nourin-Dependent miR-137 and miR-106b: Novel Biomarkers for Early Diagnosis of Myocardial Ischemia in Coronary Artery Disease Patients
}

\author{
Salwa A. Elgebaly ${ }^{1,2, *}$, Robert H. Christenson ${ }^{3}$, Hossam Kandil ${ }^{4}$, Mohsen Ibrahim ${ }^{4}$, Hussien Rizk ${ }^{4}$, \\ Nashwa El-Khazragy ${ }^{5}{ }^{(}$, Laila Rashed ${ }^{6}$, Beshoy Yacoub ${ }^{4}{ }^{\circledR}$, Heba Eldeeb ${ }^{4}$, Mahmoud M. Ali ${ }^{4}$ \\ and Donald L. Kreutzer ${ }^{2,7}$
}

check for

updates

Citation: Elgebaly, S.A.; Christenson, R.H.; Kandil, H.; Ibrahim, M.; Rizk, H.; El-Khazragy, N.; Rashed, L.; Yacoub, B.; Eldeeb, H.; Ali, M.M.; et al. Nourin-Dependent miR-137 and miR-106b: Novel Biomarkers for Early Diagnosis of Myocardial Ischemia in Coronary Artery Disease Patients. Diagnostics 2021, 11, 703. https://doi.org/10.3390/diagnostics 11040703

Academic Editor: Nicola Martinelli

Received: 24 February 2021

Accepted: 13 April 2021

Published: 14 April 2021

Publisher's Note: MDPI stays neutral with regard to jurisdictional claims in published maps and institutional affiliations.

Copyright: (c) 2021 by the authors. Licensee MDPI, Basel, Switzerland. This article is an open access article distributed under the terms and conditions of the Creative Commons Attribution (CC BY) license (https:/ / creativecommons.org/licenses/by/ $4.0 /)$.
Research \& Development, Nour Heart, Inc., Vienna, VA 22180, USA

2 Department of Surgery, UConn Health, School of Medicine, Farmington, CT 06032, USA; kreutzer@uchc.edu 3 Department of Pathology, University of Maryland School of Medicine, Baltimore, MD 21201, USA; RCHRISTENSON@umm.edu

4 Department of Cardiology, Kasr Alainy Faculty of Medicine, Cairo University, Cairo 11562, Egypt; Hossamkandil@kasralainy.edu.eg (H.K.); mibrahim_02@yahoo.com (M.I.); hussienrizk@gmail.com (H.R.); beshoy.ayuob@gmail.com (B.Y.); hebamostafakamel@gmail.com (H.E.); mahmoudbe1@hotmail.com (M.M.A.)

5 Department of Clinical Pathology-Hematology, Ain Shams Medical Research Institute (MASRI),

Faculty of Medicine, Ain Shams University, Cairo 11566, Egypt; nashwaelkhazragy@med.asu.edu.eg

6 Department of Biochemistry and Molecular Biology, Kasr Alainy Faculty of Medicine, Cairo University, Cairo 11562, Egypt; lailarashed@kasralainy.edu.eg

7 Cell \& Molecular Tissue Engineering, LLC, Farmington, CT 06032, USA

* Correspondence: selgebaly@nourheart.com; Tel.: +1-860-680-8860

Abstract: Background: Although cardiovascular imaging techniques are widely used to diagnose myocardial ischemia in patients with suspected stable coronary artery disease (CAD), they have limitations related to lack of specificity, sensitivity and "late" diagnosis. Additionally, the absence of a simple laboratory test that can detect myocardial ischemia in CAD patients, has led to many patients being first diagnosed at the time of the development of myocardial infarction. Nourin is an early blood-based biomarker rapidly released within five minutes by "reversible" ischemic myocardium before progressing to necrosis. Recently, we demonstrated that the Nourin-dependent miR-137 (marker of cell damage) and miR-106b-5p (marker of inflammation) can diagnose myocardial ischemia in patients with unstable angina (UA) and also stratify severity of ischemia, with higher expression in acute ST-segment elevation myocardial infarction (STEMI) patients compared to UA patients. Minimal baseline-gene expression levels of Nourin miRNAs were detected in healthy subjects. Objectives: To determine: (1) whether Nourin miRNAs are elevated in chest pain patients with myocardial ischemia suspected of $\mathrm{CAD}$, who also underwent dobutamine stress echocardiography (DSE) or ECG/Treadmill stress test, and (2) whether the elevated levels of serum Nourin miRNAs correlate with results of ECHO/ECG stress test in diagnosing CAD patients. Methods: Serum gene expression levels of $m i R-137, m i R-106 b-5 p$ and their corresponding molecular pathway network were measured blindly in 70 enrolled subjects using quantitative real time PCR (qPCR). Blood samples were collected from: (1) patients with chest pain suspected of myocardial ischemia $(n=38)$ both immediately "pre-stress test" and "post-stress test" $30 \mathrm{~min}$. after test termination; (2) patients with acute STEMI ( $n=16)$ functioned as our positive control; and (3) healthy volunteers $(n=16)$ who, also, exercised on ECG/Treadmill stress test for Nourin baseline-gene expression levels. Results: (1) strong correlation was observed between Nourin miRNAs serum expression levels and results obtained from $\mathrm{ECHO} / \mathrm{ECG}$ stress test in diagnosing myocardial ischemia in CAD patients; (2) positive "post-stress test" patients with CAD diagnosis showed upregulation of $m i R-137$ by 572 -fold and $m i R-106 b-5 p$ by 122-fold, when compared to negative "post-stress test" patients $(p<0.001)$; (3) similarly, positive "pre-stress test" CAD patients showed upregulation of miR-137 by 1198-fold and miR-106b-5p by 114-fold, when compared to negative "pre-stress test" patients $(p<0.001)$; and (4) healthy subjects had minimal baseline-gene expressions of Nourin miRNAs. Conclusions: Nourin-dependent miR-137 and $m i R-106 b-5 p$ are promising novel blood-based biomarkers for early diagnosis of myocardial 
ischemia in chest pain patients suspected of CAD in outpatient clinics. Early identification of CAD patients, while patients are in the stable state before progressing to infarction, is key to providing crucial diagnostic steps and therapy to limit adverse cardiac events, improve patients' health outcome and save lives.

Keywords: Nourin; stable coronary artery disease; reversible myocardial ischemia; $m i R-137$; $m i R$ 106b-5p; inflammatory biomarkers; ECHO Stress Test; ECG stress test; laboratory diagnostic test

\section{Introduction}

The incidence of coronary artery disease (CAD) is very high among different populations worldwide, and approximately 15.5 million adult persons $>20$ years have CAD [1,2]. Because of lack of a simple laboratory test that can early detect myocardial ischemia in CAD patients, many of them are being first diagnosed at the time of the development of myocardial infarction. Currently, various cardiovascular imaging techniques including dobutamine stress echocardiography (DSE) [3], exercise ECG, CT coronary angiography, and coronary angiography are the cornerstone in the diagnosis of myocardial ischemia in intermediate and high-risk groups [4,5]. However, these tests have variable sensitivity and specificity across different studies [6] and each of these tests has its own limitations [7]. Since there no blood-based biomarker exists that can diagnose myocardial ischemia in CAD patients before progressing to infarction, developing a simple laboratory test for identification of myocardial ischemia in individuals who have CAD in the stable state is key to provide crucial diagnostic steps and early therapy to limit adverse cardiac events [2].

Pathological changes during myocardial ischemia are initiated within 10 min after interruption of coronary blood-flow and the myocardium ceases its aerobic metabolism [8]. Persistence of ischemia triggers a cascade of events, starting with a decreased diastolic and later systolic function, appearance of wall motion abnormalities and finally ECG abnormalities and development of angina [9,10]. Although cardiac biomarkers such as Troponin I and $\mathrm{T}$ are the gold standard diagnostic biomarkers for myocardial infarction [11-15], challenges related to sensitivity and specificity, as well as ability to rule-out reversible ischemia, may limit their use as biomarkers for the diagnosis of CAD patients [12]. Therefore, there is a clear need to identify and validate new blood-based biomarkers to identify "reversible" ischemic injury in stable CAD patients before progressing to infarction.

Elgebaly et al. [16-25] reported that Nourin ${ }^{\circledR i s}$ a $3 \mathrm{KDa}$ formyl peptide released within five minutes of the onset of ischemia by human and animal hearts in response to ischemic injury. Nourin is unique because of its rapid release by "reversible" ischemic myocardium when cells are "injured", but still "viable" and "not dead". If ischemia persists, Nourin is also released by necrotic cells [16-25]. Nourin is a potent inflammatory mediator and its release is associated with post-ischemic cardiac inflammation in early ischemia/reperfusion animal models of AMI, cardiopulmonary bypass surgery and heart failure $[16,17,24]$. Nourin was referred to as a "cardiac-derived leukocyte chemotactic factor" and was purified from cardioplegic solutions collected during cardiac arrest ("reversible" myocardial ischemia) from patients who underwent cardiopulmonary bypass surgery for coronary revascularization, and its amino acid sequence was also determined [17,18].

Using Nourin antibody ELISA and chemotaxis assay, high levels of Nourin were detected in blood samples collected at presentation to hospital Emergency Departments (EDs) from symptomatic acute coronary symptoms (ACS) patients and patients with documented AMI within the first eight hours of chest pain. Nourin was not elevated in symptomatic non-cardiac patients and healthy subjects [25]. These results suggest the potential use of the Nourin biomarker to "rule-in" myocardial ischemia in ACS patients and "rule-out" cardiac injury for symptomatic patients having non-cardiac causes. Additionally, using the Nourin amino acid sequence, an integrated Bioinformatics analysis was recently conducted and indicated that Nourin is a direct target for miR-137 (marker of cell damage) 
and $m i R-106 b-5 p$ (marker of inflammation) in myocardial ischemic injury [21-23]. Using quantitative real time PCR (qPCR) results indicated that the Nourin-dependent miR-137 and $m i R-106 b-5 p$ can diagnose myocardial ischemia in patients with UA and also stratify severity of ischemia, with higher expression in acute STEMI patients compared to UA patients. Minimal baseline-gene expression levels of Nourin miRNAs were detected in healthy subjects [21-23].

In the present study, we tested the hypothesis that the Nourin-dependent $m i R-137$ and miR-106b-5p will be elevated in chest pain patients with myocardial ischemia suspected of CAD, who also underwent a DSE or ECG stress test, and that the elevated levels of Nourin miRNAs correlate with results of ECHO/ECG stress test in diagnosing CAD patients. As indicated in Figure 1, all chest pain patients suspected of myocardial ischemia $(n=38)$ were tested for Troponin. Patients with negative Troponin (levels below the clinical decision-99th percentile), underwent ECHO or ECG stress test. Patients with positive stress test also had significant upregulation of Nourin miRNAs both "pre-stress test" and "post-stress test", while patients with negative stress test showed baseline values of Nourin miRNAs, also "pre-stress test" and "post-stress test". Since results of this preliminary study indicate a correlation of Nourin miRNAs serum expression levels with ECHO/ECG stress test findings in determining myocardial ischemia and diagnosing CAD patients both "post-stress test" and "pre-stress test", this suggests a promising role for the novel Nourin biomarkers to "rule-in" suspected CAD patients in "outpatient clinics".

Prediction of Myocardial Ischemia in Chest Pain Patients Suspected of CAD

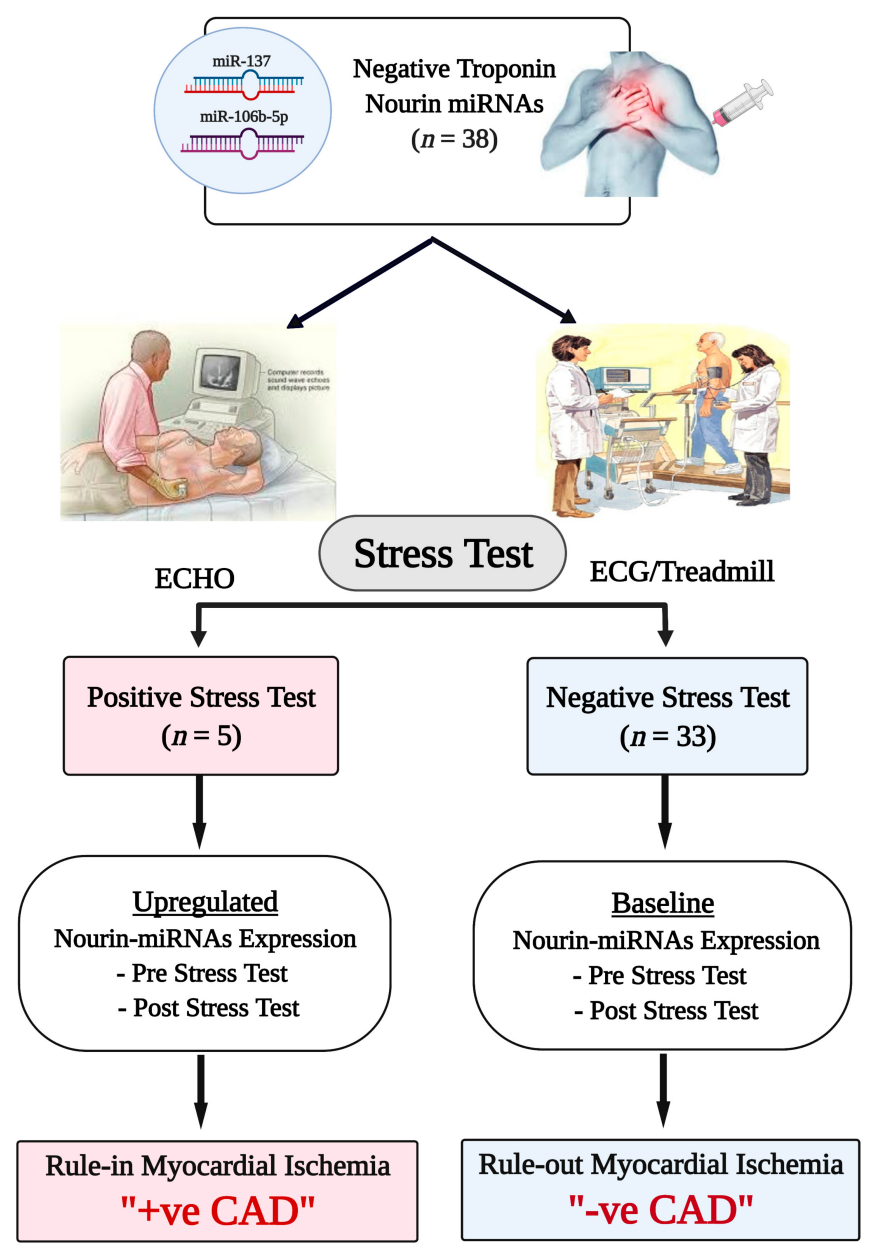

Figure 1. Flowchart Study Design. Abbreviations: CAD: coronary artery disease, ECHO: echocardiography, ECG: electrocardiogram. 


\section{Results}

\subsection{Demographic Characteristics of Participants}

The demographic and clinical characteristics of participants are presented in Table 1. Chest pain patients suspected of CAD who underwent ECHO/ECG stress test group $(n=38)$ represent the main core group of the study. Two additional control groups were enrolled: acute STEMI patients (positive control) and healthy subjects (negative control). All subjects enrolled in the study had met the inclusion/exclusion criteria and showed normal renal function (serum creatinine level $<1.2 \mathrm{mg} / \mathrm{dL}$ ). As indicated below in Tables 1 and 2, five out of the 38 chest pain patients showed positive ECHO/ECG stress test, while the rest of the 33 patients showed negative stress test. Because of reagent limitations, serum samples of only seven patients out of the 33 negative stress test patients were used to determine the levels of Nourin molecular network.

Table 1. Baseline Clinical Characteristics of Different Patient Groups.

\begin{tabular}{|c|c|c|c|c|c|}
\hline \multirow[b]{2}{*}{ Variable } & \multirow{2}{*}{$\begin{array}{l}\text { Healthy } \\
(n=16)\end{array}$} & \multicolumn{2}{|c|}{ ECHO/ECG Stress Test } & \multirow{2}{*}{$\begin{array}{l}\text { STEMI } \\
(n=16)\end{array}$} & \multirow[b]{2}{*}{$p$-Value } \\
\hline & & $\begin{array}{c}\text { Negative } \\
(n=7)\end{array}$ & $\begin{array}{c}\text { Positive } \\
(n=5)\end{array}$ & & \\
\hline Age (Years) Mean \pm SD & $32.9 \pm 9.9$ & $53.5 \pm 9.9$ & $55 \pm 4.8$ & $54.4 \pm 12.7$ & 0.001 \\
\hline Sex: Males: $n(\%)$ & $16(100)$ & $3(43)$ & $4(80)$ & $12(75)$ & 0.014 \\
\hline \multicolumn{6}{|l|}{ Risk Factors } \\
\hline BMI $\left(\mathrm{kg} / \mathrm{m}^{2}\right)$ Mean & $26.8 \pm 4.7$ & 31.0 & 30.0 & $31.4 \pm 4.8$ & 0.145 \\
\hline Smoking: $n(\%)$ & $8(50)$ & $2(29)$ & - & $10(52.5)$ & 0.09 \\
\hline Diabetes Mellitus: $n(\%)$ & - & $2(40)$ & $5(71)$ & $6(37.5)$ & 0.15 \\
\hline Hypertension: $n(\%)$ & - & $4(57)$ & $2(40)$ & $7(43)$ & 0.82 \\
\hline Dyslipidemia: $n(\%)$ & - & $3(43)$ & $2(40)$ & $5(31)$ & 0.6 \\
\hline
\end{tabular}

ECHO: echocardiography, ECG: electrocardiogram, STEMI: ST-segment elevation myocardial infarction, BMI body mass index, $n$ : number of cases, \%: percentage of cases calculated as percentage in the group, SD: standard deviation, $p$-value $>0.05$ considered statistically insignificant, $p$-value $<0.05$ considered statistically significant.

\subsection{Nourin Network Gene Expression Profiles in Patients Suspected of Myocardial Ischemia}

Chest pain patients suspected of myocardial ischemia $(n=38)$ underwent standard stress tests using dobutamine stress echocardiography (DSE) or exercise ECG/Treadmill stress test for the diagnosis of stable CAD. The Nourin-dependent miRNAs and their related molecular network genes were measured blindly in serum samples collected immediately "pre" the DSE or exercise ECG stress test, as well as $30 \mathrm{~min}$ "post" termination of the test from symptomatic patients suspected of myocardial ischemia. As indicated below, five out of the 38 chest pain patients showed positive ECHO/ECG stress test, while the rest of the 33 patients showed a negative stress test. Because of reagent limitations, serum samples of only seven patients out of the 33 negative stress tests patients, were used to determine the levels of Nourin molecular network.

\subsubsection{Assessment of CTB89H12.4/miR-137/FTHL-17 Network "Post-Stress Test"}

The expression levels of Nourin-dependent genes were measured blindly in serum samples of outpatients with chest pain suspected of myocardial ischemia using qPCR. The assessment was performed $30 \mathrm{~min}$ after exercise-induced ischemia (stress test). Upregulation of $m i R-137$ and FTHL-17, which are significantly associated with induced ischemia, are reflected by high expression levels post-stress test as presented in Table 2 and Figure 2. Specifically, Nourin-dependent miR-137 and FTHL-17 gene expression levels were significantly correlated with results obtained from ECHO or exercise ECG stress tests in determining the presence or absence of myocardial ischemia. As indicated in Figure 2, miR-137 (Figure 2a) was upregulated by 572-fold and 69-fold for FTHL-17 (Figure 2b) in serum expression of patients with positive stress test compared to negative stress test patients $(p=0.001)$, results which are consistent with the positive ECHO and ECG findings. Furthermore, when gene expression levels were compared in negative stress test patients 
to healthy controls, results showed higher expression levels of $m i R-137$ (4.2-fold, $p=0.01$ ) (Figure 2a) in negative stress test compared to healthy, while for FTHL-17, there was higher gene expressions (2-fold, $p=0.01$ ) (Figure $2 \mathrm{~b}$ ) in healthy subjects compared to negative stress test patients. Additionally, when gene expression levels were compared between acute STEMI patients and patients with positive stress test, a statistically significant difference was detected for the expression of $m i R-137$ (1.22-fold, $p=0.01$ ) (Figure 2a), while FTHL-17 was reduced by 2.6-fold in acute STEMI patients compared to positive stress test $(p=0.01)$ (Figure $2 \mathrm{~b}$ ). The median expression level of the candidate genes and the fold changes are presented in Table 2.

Table 2. Post-ECHO/ECG stress test gene expression profiles of chest pain patients with positive and negative stress test versus healthy (negative controls) and STEMI (positive controls).

\begin{tabular}{|c|c|c|c|c|}
\hline \multirow[b]{2}{*}{ Variable } & \multirow{2}{*}{$\begin{array}{c}\begin{array}{c}\text { Negative } \\
\text { Control }\end{array} \\
\text { Healthy } \\
(n=16) \\
\text { Median } \\
\text { (min-max) }\end{array}$} & \multicolumn{2}{|c|}{ Post-ECHO/ECG Stress Test } & \multirow{2}{*}{$\begin{array}{l}\text { Positive Control } \\
\text { STEMI } \\
(n=16) \\
\text { Median } \\
(\text { min-max) }\end{array}$} \\
\hline & & $\begin{array}{c}\text { Negative } \\
(n=7) \\
\text { Median } \\
\text { (min-max) }\end{array}$ & $\begin{array}{c}\text { Positive } \\
(n=5) \\
\text { Median } \\
\text { (min-max) }\end{array}$ & \\
\hline miR-137 $\left(\log _{10}\right)$ & $1.05(0.1-3.7)$ & $4.5(1.5-5.0)$ & $2575(917-3774)$ & 3163 (936-9878) \\
\hline miR-106b $\left(\log _{10}\right)$ & $1.08(0.2-3.6)$ & $3.3(1.8-8.5)$ & $512(305-803)$ & $953(307-2504)$ \\
\hline FTHL-17 $\left(\log _{10}\right)$ & $3.8(1.6-16.7)$ & $1.9(0.2-4.6)$ & $131(49-228)$ & $50(33-76)$ \\
\hline $\begin{array}{l}\text { ANAPC11 } \\
\left(\log _{10}\right)\end{array}$ & $0.9(0.1-3.9)$ & $0.2(0.15-1.7)$ & $11.9(5.4-18.0)$ & $8.4(4.5-25.0)$ \\
\hline $\begin{array}{l}\text { СТВ89H12.4 } \\
\left(\log _{10}\right)\end{array}$ & $65(26-83)$ & $0.8(0.5-1.1)$ & $0.07(0.02-0.1)$ & $0.9(0.14-3.2)$ \\
\hline
\end{tabular}

ECHO: echocardiography, ECG: electrocardiogram, STEMI: ST-segment elevation myocardial infarction, FTHL-17, Ferritin heavy chain like 17, ANAPC11: anaphase promoting complex subunit, CTB89H12.4 is a long non-coding RNA (lncRNA), min: minimum, and max: maximum

\subsubsection{Assessment of CTB89H12.4/miR-106b-5p/ ANAPC11 Network" Post-Stress Test"}

The serum expression levels of Nourin-dependent miR-106b-5p and ANAPC11 were measured blindly in serum samples of patients with chest pain suspected of myocardial ischemia. The assessment was performed $30 \mathrm{~min}$ after exercise-induced ischemia (stress test). There was an upregulation of $m i R-106 b-5 p$ and ANAPC11 post-stress test which are significantly correlated with results obtained from ECHO or exercise ECG stress tests in determining the presence or absence of myocardial ischemia. As indicated in Table 2 and Figure 2, serum levels of miR-106b-5p (Figure 2d) and ANAPC11 (Figure 2e) were significantly increased by 122 -fold and 60-fold, respectively, in patients with positive stress test ( $p=0.0001)$ compared to negative stress test patients, results which are consistent with the ECHO and ECG findings. In addition, higher miR-106b-5p (Figure 2d) (three-fold increase, $p=0.001$ ) was detected in patients with negative stress test compared to healthy controls $(p<0.01)$, while for ANAPC11 (Figure 2e), there were higher gene expressions (4.5-fold, $p=0.04$ ) (Figure 2e) in healthy subjects compared to negative stress test patients. Furthermore, when gene expression levels were compared between acute STEMI patients and patients with positive stress test, there was no statistically significant difference for gene expression of miR-106b-5p (1.8-fold increase, $p=0.06$ ) and ANAPC11 (1.4-fold increase, $p=0.06$ ) (Figure $2 \mathrm{~d}$ ). The median expression level of the candidate genes and the fold changes are presented in Table 2.

Gene expression level of $\operatorname{lnc}-\mathrm{CTB} 89 \mathrm{H} 12.4$ tends to be downregulated in ischemic injury. Contrary to the upregulation of miR-137, FTHL-17, $m i R-106 b-5 p$ and ANAPC11, a significant reduction level of $\operatorname{lnc}-\mathrm{CTB} 89 \mathrm{H} 12.4$ by 11.4 -fold was detected in serum of patients with chest pain who were positive to stress test compared to negative stress test patients $(p<0.01)$ (Figure 2c). Similarly, a significant difference was detected between patients with negative stress test and healthy subjects $(p=0.0001)$, and between STEMI patients 
compared to patients with positive stress test $(p=0.0001)$ (Figure 2c). Additionally, serum lnc-CTB89H12.4 expression was decreased by 81-fold in patients with negative stress test compared to healthy controls (Figure 2c), and by 12.8-fold in STEMI patients compared to patients with positive stress test $(p<0.0001)$ (Figure $2 \mathrm{c}$ ), respectively. The median expression levels of genes are presented in Table 2.
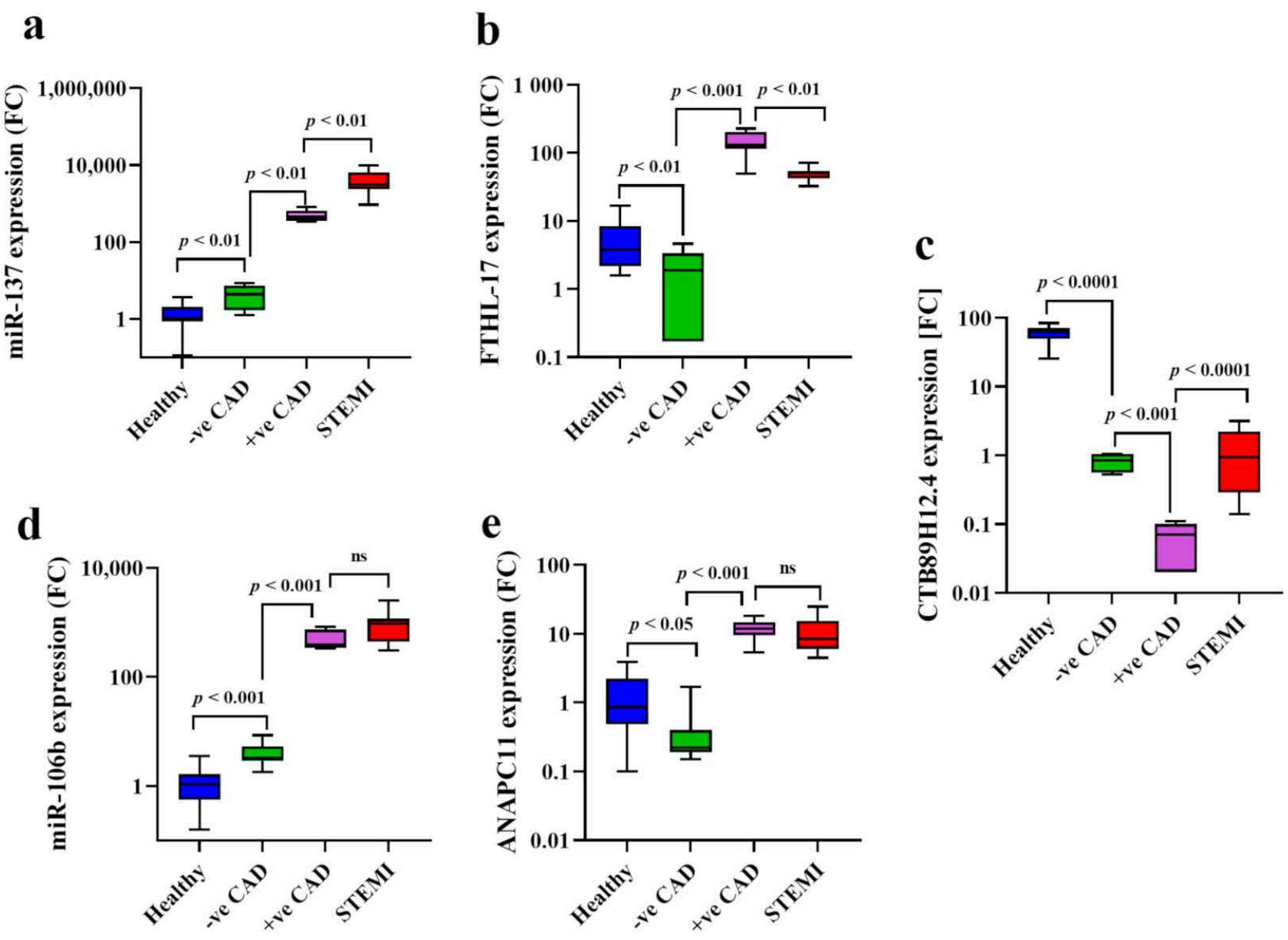

Figure 2. Boxplot graphs demonstrating the serum expression levels of $m i R-137$ in patients with chest pain and suspected for CAD; higher expression levels of miR-137 (a), FTHL-17 (b), miR-106b (d) and ANAPC11 (e) were significantly associated with CAD; lnc-CTB89H12.4 was downregulated (c); Abbreviations: CAD: coronary artery disease, STEMI: ST-segment elevation myocardial infarction, FTHL-17: Ferritin heavy chain like 17, ANAPC11: anaphase promoting complex subunit, $p<0.05$ considered significant, ns: no-significant difference.

\subsection{Assessment of Nourin-Dependent miR-137 and miR-106b Gene Expression Profiles "Pre-Stress Test" and "Post-Stress Test" (30 Minutes After Termination) in Chest Pain Patients Suspected of Myocardial Ischemia}

Gene expression profiles of $m i R-137$ and $m i R-106 b-5 p$ were compared in "pre-stress test" and "post-stress test" samples of positive and negative stress test patients in order to: (1) determine whether Nourin miRNAs are detected in blood circulation before stress-induced ischemia, and (2) evaluate the potential clinical use of Nourin miRNAs in diagnosing chest pain patients suspected of myocardial injury in outpatient clinics. Results indicated marked upregulation by 1198-fold (median: 2157) in miR-137 expression level in "pre-stress test" patients with positive stress test compared to negative stress test patients (median: 1.8) $(p<0.001)$ (Figure 3a). Similar results were obtained for $m i R-106 b-5 p$ with upregulation by 114-fold (median: 423.2) in "pre-stress test" patients with positive stress test compared to negative stress test patients (median: 3.7) ( $p=0.01)$ (Figure 3c). Furthermore, gene expression patterns for Nourin miR-137 (Figure 3b) indicated no sta- 
tistical significance was reached $(p>0.05)$ between "pre-stress test" and "post-stress test" samples in patients with positive and negative stress test. However, there was a slight elevation of the inflammatory marker, $m i R-106 b-5 p$ (Figure 3c) in patients with positive stress test ( $p=0.02)$ detected "post-stress test" (median: 512.0) compared to "pre-stress test" (median: 423.2), which may likely reflect an ultimate association between miR-106b-5p and inflammatory cytokines associated with ischemia-induced aggravation and tissue hypoxia.
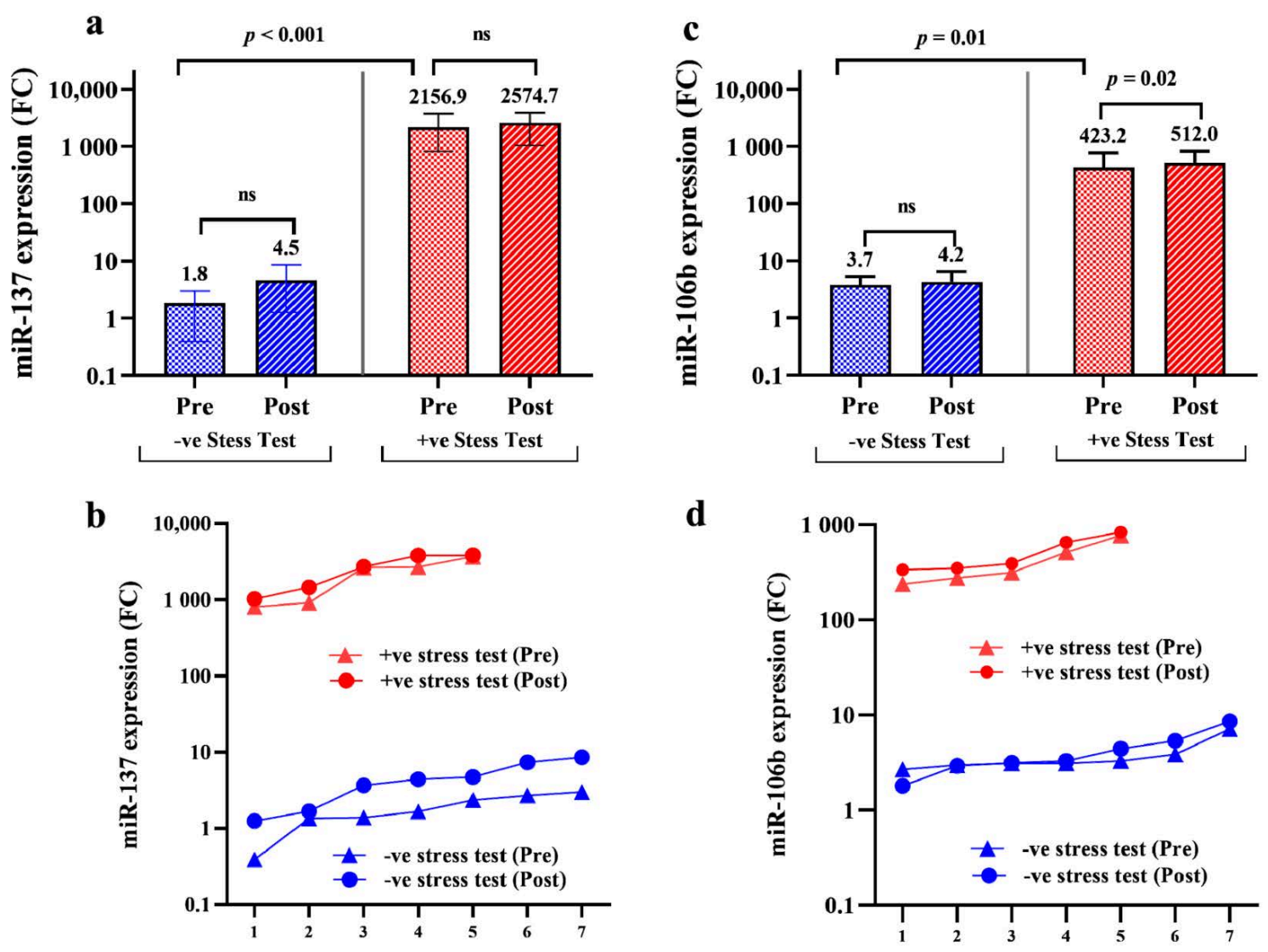

Figure 3. Bar chart graph illustrates $m i R-137$ (a) and $m i R-106 b-5 p(\mathbf{b})$ expression levels in patients with chest pain and suspected of CAD; miRNA expression levels were measured before stress test-induced ischemia, "pre-stress test" and 30 min "post-stress test"; the "expression patterns" of Nourin miRNAs, miR-137 (c) and miR-106b-5p (d) were plotted for positive stress test patients "pre-stress test" and $30 \mathrm{~min}$ "post-stress test" and compared to patients who showed negative results in order to investigate the computability of Nourin miRNAs level and stress test results; $p<0.05$ considered significant; ns: no-significant difference.

\section{Discussion}

Despite the progress made in developing diagnostic tests to identify patients with stable $C A D$, there are still challenges and obstacles facing clinicians in diagnosis for this patient population without resorting to invasive interventions [2]. Currently, diagnosis of stable CAD depends on detecting ischemia by different stress test modalities or by detecting significant coronary artery stenosis using CT coronary angiography. However, these tests have variable sensitivity and specificity across different studies and some limitations such as radiation exposure and contrast use in CT coronary angiography [26], or suboptimal echocardiographic window in dobutamine stress echocardiography $[27,28]$. One approach to overcome these limitations is the use of blood-based biomarkers of myocardial ischemia for early diagnosis of CAD.

Recently, many efforts have been focused on exploring the development of early, simple, sensitive and non-invasive diagnostic blood-based biomarkers for CAD in order to provide crucial therapy to reduce the progression of the disease and lower the mortality 
rate [29]. However, measuring biomarkers in individuals with suspected CAD who underwent a stress test showed variable results across different studies. Highly-sensitive cardiac Troponin (hs-cTn) is one of the most commonly studied biomarkers to diagnose CAD, but Troponin level before stress test had low sensitivity in diagnosing CAD [30]. Wongpraparut et al. [31] reported that elevated Troponin level had 43\% sensitivity in predicting moderate to severe ischemia by pharmacological stress test. Another study by Suchlez et al. [32] reported that elevated Troponin level had 53\% sensitivity in detecting myocardial ischemia in patients with significant coronary artery stenosis diagnosed by coronary angiography. Meanwhile, Braunwald et al. [33] indicated that there is a quantifiable rise in Troponin level in patients who developed myocardial ischemia during exercise stress test and that the magnitude of this rise was proportional to the degree of ischemia on perfusion imaging. However, other studies demonstrated that inducible ischemia did not change Troponin level $18 \mathrm{~min}$. or $4 \mathrm{~h}$ after the end of stress test [34]. Furthermore, since the rising pattern of Troponin after stress test is variable with inconsistent results in different studies [35], it represents a limitation that affects the diagnostic and prognostic value of Troponin for CAD patients [32].

MicroRNAs (miRNAs) have been illustrated to regulate different cellular biological processes and regulate gene expression, therefore they are superior to protein for early diagnosis of inflammatory and cardiac diseases. Recently, circulating miRNAs have been established as new markers of disease, including cardiac injury and inflammation $[13,21-23,29,36-38]$. Previous studies have demonstrated the diagnostic potential of exosomal miRNAs such as $m i R-149-5 p, m i R-942-5 p, m i R-133 b, m i R-21$ and $m i R-32-5 p$ in stable CAD patients $[39,40]$. Additionally, a panel of circulating microRNAs were found to serve as early diagnostic biomarkers for stable CAD [41], whereas higher expression levels of miR-765, miR-483, miR-122 and miR-155 were demonstrated early, but miR-149 and miR-451a were downregulated [42] in these stable CAD patients.

Using the Nourin amino acid sequence, we conducted an integrated bioinformatics analysis which indicated that Nourin molecular network is composed of $\operatorname{lncRNA}$ CTB89H12.4, miR-137, miR-106b-5p, mRNA-FTHL-17, and mRNA-ANAPC11 and that they are an autophagy-related RNA-based network linked to each other and to cardiovascular ischemia. Recently, Elgebaly et al. [21-23] reported that the Nourin-dependent miR-137 was up-regulated by 1185-fold in unstable angina patients compared to healthy volunteers, and by 2.5-fold in acute STEMI patients compared to UA patients. Similarly, the Nourin-dependent $m i R-106 b$ - $5 p$ was up-regulated by 150-fold in UA compared to healthy volunteers and by 4.6-fold in acute STEMI compared to UA patients. Unstable angina patients were confirmed by invasive coronary angiography, where all patients showed stenosis greater than $50 \%$ and Troponin level below the clinical decision limit. Baseline values of $m i R-137$ and $m i R-106 b-5 p$ were detected in healthy subjects. Furthermore, receiver operator characteristics (ROC) analysis revealed that the two miRNAs were sensitive and specific biomarkers for assessment of UA and STEMI patients [21]. We also reported that miR-137 and miR-106b-5p were significantly correlated to FTHL-17 and ANAPC11, respectively, while $\operatorname{lnc}-\mathrm{CTB} 89 \mathrm{H} 12.4$ was negatively correlated to Nourin-dependent $m i R-137$ and miR-106b-5p [21-23]. Elgebaly et al. [24] also, reported that Nourin miRNAs were significantly upregulated in the standard Isoproterenol (ISO) rat model of heart failure (HF) induced by demand ischemia. Gene expression profiles showed upregulation of miR-137 and $m i R-106 b-5 p$ by 8.6-fold and 8.7-fold, respectively, in the heart failure of ISO/saline rats compared to control/saline group. Interestingly, treating ISO "HF rats" with our bioenergetic drug, Cyclocreatine Phosphate (CCrP) prevented ischemic injury and the development of heart failure, resulting in normal ejection fraction and physical activity $[16,19]$. CCrP treatment also markedly reduced gene expression of miR-137 by $75 \%$ and of $m i R-106 b-5 p$ by $44 \%$ in the ISO/CCrP rats, "non-HF rats," compared to ISO/Saline rats, "HF rats" [24]. Consistent with our results, miR-137 was reported by others to be linked to ischemic injury, where $m i R-137$ exacerbates apoptosis of ischemia-reperfusion injured cardiomyocyte [43]. Recently, miR-106b-5p was reported to be implicated in pathogenesis 
of myocardial ischemic injury, where higher expression level of miR-106b-5p was demonstrated in cardiomyocyte cells immediately after exposure to hypoxia and apoptosis [44].

In the current study, we evaluated: (1) whether the Nourin-dependent miRNA network can detect myocardial ischemia in chest pain patients suspected of CAD, and (2) whether the elevated levels of Nourin miRNAs in CAD patients correlate with the positive diagnosis of myocardial ischemia by dobutamine stress echocardiography or exercise ECG tests. Additionally, we compared gene expressions of Nourin-dependent miRNAs in stable CAD patients to levels detected in acute STEMI patients (Nourin positive control) and healthy subjects (Nourin negative control). A total of 70 subjects who met the inclusion/exclusion criteria were enrolled in this study. Thirty-eight $(n=38)$ consecutive chest pain patients, in whom CAD was suspected, underwent DSE or ECG stress test. "Pre-stress test" and "post-stress test" of circulating miR-137 and miR-106b-5p expression levels were tested blindly in patient's sera using quantitative real time PCR (qPCR). Additionally, miRNAs targets genes (FTHL-17 and ANAPC11) and lncRNA regulatory gene (CTB89H112.4) were tested in parallel to the miRNAs. Results indicated that a strong correlation was observed between Nourin miRNAs serum expression levels and results from ECHO/ECG stress test in diagnosing myocardial ischemia in CAD patients. There was a significant elevation of Nourin-dependent $m i R-137$ and $m i R-106 b-5 p$ in patients with inducible myocardial ischemia by stress test (positive CAD) compared to symptomatic patients with no inducible myocardial ischemia (negative CAD). Specifically, "post-stress test" positive CAD patients showed upregulation of miR-137 by 572 -fold increase and $m i R-106 b-5 p$ by 122 -fold when compared to negative test patients $(p<0.001)$. Similarly, "pre-stress test" showed upregulation of miR-137 by 1198 -fold and miR-1066-5p by 114 -fold in comparison to negative test patients $(p<0.001)$.

The observed high miRNA level "pre-stress test" can possibly be explained by the continuous steady-state release of Nourin into the circulation in response to the chronic myocardial ischemia state in CAD patients. It is likely that the small size of Nourin ( $3 \mathrm{kDa})$ facilitates its transport across cell membranes from viable ischemic cardiomyocytes leading to its continuous presence in blood circulation before induction of ischemia. Although CAD patients continued to show elevation of Nourin miRNAs after inducible myocardial ischemia, there was no significant difference in the elevated levels of $m i R-137$, as a marker of "cell damage" both "pre-stress test" and "post-stress test" $(p<0.275)$. However, there was a slightly higher levels of the "inflammatory" marker, miR-106b-5p, "post-stress test" compared to "pre-stress test" $(p=0.02)$, suggesting a role of ischemia-induced inflammation. Healthy subjects' sera collected "post-stress test" showed minimal baseline-gene expression levels of Nourin miRNAs.

It has been reported that the prevalence of myocardial ischemia in patients with intermediate pre-test probability for obstructive CAD is only $14 \%$ [45,46]. Although in our study the small sample size of CAD patients $(n=5)$ is a limitation, it is attributed to the small percentage of patients in whom the stress test turned to be positive for myocardial ischemia among the total number of patients $(n=38)$. The 5 CAD patients constitute $13 \%$, which is comparable to published reports [45,46], while for the remaining 33 negative patients, for four of the test was inconclusive and 29 had normal stress test. Larger clinical studies are needed to determine the potential clinical utility of the Nourin gene-based test in identifying myocardial ischemia in CAD patients.

\section{Subjects \& Methods}

\subsection{Bioinformatics Analysis}

Bioinformatics analysis was conducted to identify the differentially expressed genes (DEGs) that are related to Nourin amino acid sequence and also linked to myocardial ischemic injury, as we previously reported [21]. Briefly, three genes' expression profiles were downloaded from the Gene Expression Omnibus (GEO) database and integrated Bioinformatics analysis was applied. Initially, the functional gathering analysis was used to map the Nourin protein-genes interaction using the Kyoto Encyclopedia of Genes 
and Genomes (KEGG), as well as incorporated gene ontology (GO) databases. In order to explore the biological characteristics of the DEGs, an interaction network of proteinprotein internet (PPI) was created using the STRING database. Two upregulated genes named "Ferritin heavy chain like 17 (FTHL-17) and Anaphase Promoting Complex Subunit (ANAPC11)" were selected based on the following: (1) direct targets of Nourin protein, (2) expressed on normal myocardium tissue, and (3) significantly dysregulated in ischemic injury. Subsequently, we constructed the $\operatorname{lnc} R N A / m i R N A / m R N A$ networks to predict the upstream regulating genes of Nourin. Using the miRDB, TargetScan and miRTarBase databases, two miRNAs "miR-137 and miR-106b" were found to regulate the expression of FTHL-17 and ANAPC11 genes, respectively. Furthermore, Starbase and CHIP, as well as DIANA-LncBasev3

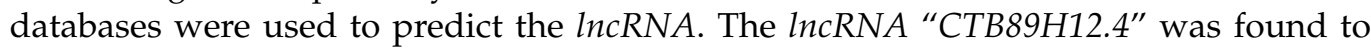
regulate the expression of both $m i R-137$ and $m i R-106 b$. To ensure accuracy of the obtained data, we used three different databases to identify the high complementarity binding sites between different genes and Nourin protein. Finally, each network " $[F T H L-17 / m i R-137 / \operatorname{lnc}$ CTB89H12.4] and [ANAPC11/miR-106b/lnc-CTB89H12.4]" was verified using the Clustal 2 multiple sequence alignment software for the validation of the gene-gene alignment.

\subsection{Study Design and Protocol}

4.2.1. Study Participants

A total of 70 subjects who met the inclusion/exclusion criteria were enrolled in the study between October 2019 and March 2020 and they were categorized into three groups: (1) patients complaining of chest pain suspected of CAD with negative Troponin I, and who underwent DSE or exercise ECG tests to "rule-in" or "rule-out" the presence of significant myocardial ischemia $(n=38)$; (2) patients presented with acute ST elevation myocardial infarction in the first $10 \mathrm{~h}$ from onset of symptoms with positive Troponin I or highlysensitive Troponin T (hs-TnT) $(n=16)$; and (3) control group of healthy volunteers $(n=16)$ with normal physical examination and no history of cardiac or other system disease, who also underwent exercise ECG testing to confirm negative myocardial ischemia. The study was conducted according to the principles outlined in the Declaration of Helsinki, and approved by the Ethics Community of the Faculty of Medicine, Cairo University, Cairo, Egypt (Approval MS-62-2019). All patients provided a written, informed consent before enrollment. The study workflow is illustrated in Figure 1.

\subsubsection{Study Protocol}

Detailed medical history was obtained from all patients submitted to the study and the presence of any of the conventional coronary artery disease risk factors was recorded, including age, diabetes mellitus, hypertension, hypercholesterolemia, smoking, previous history of coronary artery disease, myocardial infarction, percutaneous coronary intervention (PCI), and coronary artery bypass grafting (CABG). Apart from patients with acute STEMI, patients were excluded if they had active symptoms of myocardial ischemia or infarction, (within the last month), history of recent MI or PCI, positive Troponin I or T, positive C-reactive protein (CRP), history of cardiomyopathy or heart failure or congenital heart disease, end stage renal failure, hepatitis or hepatic failure, history of thoracic irradiation therapy, malignant diseases, autoimmune diseases and inflammatory diseases such as inflammatory bowel disease and arthritis, as well as contraindications to stress testing of various causes according to the European Association of Echocardiography (EAE) Expert Consensus Statement [45].

\subsection{Dobutamine Stress Test Protocol}

\subsubsection{Dobutamine Stress Echocardiography}

For this study, we used the European Association of Echocardiography Expert Consensus Statement: An iE33 (Philips Medical Systems, Andover, MA, USA) ultrasound machine [45] Studies were performed by an experienced operator. Dobutamine was intravenously infused during 3-min stages at incremental doses of 10, 20, 30, and $40 \mu \mathrm{g} / \mathrm{kg} / \mathrm{min}$ 
until $\geq 85 \%$ of the age-predicted maximal heart rate was reached. ECG and symptoms were monitored continuously and blood pressure was measured every $3 \mathrm{~min}$. If the target heart rate was not achieved at the peak dose of dobutamine infusion, Atropine was administrated in $0.25 \mathrm{mg}$ increments to a maximal dose of $2.0 \mathrm{mg}$. Stress testing was discontinued when the target heart rate was achieved or when one of the following events occurred: (1) new wall motion abnormality in $\geq 2$ adjacent segments, severe chest pain or dyspnea, $\geq 40 \mathrm{mmHg}$ decrease in systolic blood pressure, severe arterial hypertension of $\geq 220 / 120 \mathrm{mmHg}$ or life threatening arrhythmia [45].

\subsubsection{Stress ECG Protocol}

The stress ECG was performed on a treadmill according to the Bruce protocol. A standard 12-lead ECG was recorded regularly prior to, during every stage level, and after the exercise rest. The test was interpreted by two expert cardiologists. An interpretation of the test included the duration of exercise, peak treadmill speed and grade, maximum heart rate and percentage of age-predicted maximal heart rate achieved "220-age (years)", resting and peak blood pressure, functional capacity, ST-segment changes, and occurrence of arrhythmias.

Clinical findings that suggest an exercise-stress test "positive" for ischemia include exercise-induced hypotension, exercise-induced angina or anginal equivalents with significant ST-segment changes (defined by either $\geq 0.1 \mathrm{mV}$ horizontal or down-sloping depression or $\geq 0.1 \mathrm{mV}$ ST-segment elevation $60-80 \mathrm{~ms}$ after the J-point at the end of the QRS time), malignant arrhythmias, and/or appearance of an S3, S4 or heart murmur during exercise. A "negative" test result was the lack of any of the above-mentioned findings. Stress test termination criteria were defined as relevant ST-segment changes, angina pectoris, severe dyspnea and exhaustion, severe hypertension (>220 $\mathrm{mmHg}$ systolic), hypotension and life-threatening arrhythmias [45].

\subsection{Gene Amplification Analysis}

Three mL blood samples were collected from patients at the presented scheduled time, as follows: (1) for STEMI patients, samples were collected immediately at presentation to hospital ED; (2) for healthy subjects, samples were collected 30 min after ECG-treadmill stress test; and (3) for the stress test group, two blood samples were collected immediately before test and $30 \mathrm{~min}$ after termination of dobutamine echocardiography and exercise ECG tests. To obtain the sera, the blood samples were centrifuged at $1300 \times g$ at $4{ }^{\circ} \mathrm{C}$ for $20 \mathrm{~min}$, and the collected sera was stored at $-80{ }^{\circ} \mathrm{C}$ until analyzed.

Total RNAs and miRNAs were extracted from subjects' sera using the RNeasy Mini Kit (Qiagen, Hilden, Germany). After spectrophotometric measurement of the RNA concentration and purity, the cDNA was synthesized by reverse transcription reaction using miScript RT Kit (Qiagen, Hilden, Germany). Subsequently, the expression profiles of the five genes " $m i R-137$, miR-106b, FTHL-17, ANAPC11 and lnc-CTB89H12.4" were measured using quantitative Real time PCR (qPCR). The specific primer sequence assays including Hs_miR-137_1,Hs_miR-106b-5p_1,Hs_FTHL17_1,Hs_ANAPC11_1, and RT ${ }^{2}$ CTB89H12.4], and also Hs_RNU6-2_11 and Hs_GAPDH genes, which were used as reference housekeeper gene, were purchased from (Qiagen, Hilden, Germany). Following gene amplification, the gene expression was calculated in each sample using the $2^{\Delta \Delta \mathrm{Ct}}$ analysis. All PCR procedures were conducted according to the manufacture instructions. PCR was conducted on Rotor-Gene Q 5plex HRM Platform (Qiagen, Hilden, Germany).

\subsection{Troponin Measurement}

All subjects including healthy volunteers underwent analysis of cardiac Troponin before the stress test using Elecsys Troponin I assay (Cobas e601 Analyzer, Roche Diagnostics), with the 99th percentile upper reference limit as $400 \mathrm{ng} / \mathrm{L}$, as well as hs-Troponin $\mathrm{T}$ assay (Cobas 6000 Analyzer, Roche Diagnostics) with the 99th percentile upper reference limit as $5 \mathrm{ng} / \mathrm{L}$. 


\subsection{Statistical Analysis}

Statistical analysis was performed using GraphPad Prism software, (version no: 8.0.2; GraphPad Software Inc.). The median value and minimum to maximum range were used to present the skewed data, while mean $\pm \mathrm{SD}$ were used for normally distributed numerical data, number of cases and percentage using cross-tabulation. Since, the gene expression levels are not normally distributed among groups, the comparative analysis between two groups was performed using the non-parametric Mann-Whitney U test. However, ANOVA was used to evaluate the difference in gene expression for more than two groups. The Wilcoxon signed-rank test was used to test the significant difference for gene expression between paired samples ("pre- and post-stress test"). In addition, we compared gene expression levels between the "pre-stress test" groups (negative vs positive) using the Welch's t test. Significance was set at $p \leq 0.05$.

\section{Conclusions}

Although Troponin helps accurate and timely diagnosis of acute (thrombotic) myocardial infarction, it is not useful in the absence of myocardial injury, even with ongoing ischemia, and it cannot confirm the diagnosis of symptomatic or silent ischemia during stress testing [30-35]. Small studies have indicated a possible incremental value of Troponin in diagnosing $C A D$, but larger trials are needed to verify the utility of systematic assessment in patients suspected of $\mathrm{CAD}$, since biomarkers do not yet have a role in diagnosing obstructive CAD [47].

Recent gene expression testing provides a new approach for blood testing with a relatively high sensitivity and negative predictive value for CAD in symptomatic patients, but it is not widely available and has not been evaluated in asymptomatic patients [48] Therefore, developing a simple laboratory test, like Nourin, for early identification of myocardial ischemia in individuals who have $\mathrm{CAD}$ in the stable state before progressing to infarction is key to providing crucial diagnostic steps to limit adverse cardiac events. Nourin-dependent miR-137 and miR-106b-5p have the diagnostic potential to "rule-in" myocardial ischemia in symptomatic patients who are suspected of CAD with negative Troponin. The ability of Nourin miRNAs to diagnose myocardial ischemia in these suspected CAD patients was consistent with results obtained from DSE or exercise ECG stress tests. Furthermore, the observed elevated levels of Nourin miRNAs "pre-stress test" might be used as an early "diagnostic" test in outpatient clinics to "rule-in" patients with suspected $\mathrm{CAD}$, thus providing crucial therapy to improve patients' health outcome and save lives.

Additionally, although percutaneous coronary intervention (PCI) is widely used to treat patients with $\mathrm{CAD}$, the procedure is receiving much criticism because of the limited morbidity and mortality benefits for some stable CAD patients [49]. A major limitation is the lack of a simple blood test that can verify patients with large areas of severe ischemic myocardium in the stable subset of patients with coronary artery disease, whom would likely benefit from the PCI procedure [50]. Despite our encouraging preliminary data, the small sample size is a major limitation of the current analysis and all our results should be considered as hypothesis-generating. Larger clinical studies are needed to determine whether the Nourin gene-based test: (1) can identify CAD patients with severe myocardial ischemia whom will be likely to benefit from PCI before conducting the procedure, (2) is a novel diagnostic biomarker to identify early-stage myocardial ischemia not only in symptomatic CAD patients, but also in asymptomatic, and (3) can predict severity of myocardial ischemia (mild, moderate and severe) in CAD patients.

Author Contributions: Conceptualization, S.A.E.; methodology, S.A.E., R.H.C., H.K., M.I., H.R., N.E.-K., L.R., B.Y., H.E., M.M.A., and D.L.K.; writing—original draft preparation, S.A.E. and N.E.-K.; writing-review and editing, S.A.E., R.H.C., H.K., M.I., H.R., N.E.-K., L.R., B.Y., H.E., M.M.A., and D.L.K.; supervision, S.A.E., M.I., and N.E.-K.; formal analysis, S.A.E. and N.E.-K.; funding acquisition, S.A.E. and D.L.K.; All authors have read and agreed to the published version of the manuscript. 
Funding: This study was supported by Nour Heart, Inc., Vienna, VA 22180, USA and Cell and Molecular Tissue Engineering LLC, Avon, CT 06001, USA.

Institutional Review Board Statement: The study was conducted according to the guidelines of the Declaration of Helsinki, and approved by the Research Ethics Committee, Faculty of Medicine, Cairo University, Egypt, dated 12 October 2019.

Informed Consent Statement: Informed consent was obtained from all subjects involved in the study.

Data Availability Statement: The data presented in this study are available on request from the corresponding author. The data presented in this study are available on request from the corresponding author. The data are not publicly due to privacy.

Conflicts of Interest: S.A.E. Elgebaly is the founder of Nour Heart, Inc. and R. H. Christenson, reports receiving a research grant from Spingotech Diagnostics, Roche Diagnostics, Siemens Diagnostics, Becton Dickinson. D.L.K. Kreutzer is the co-founder of Cell and Molecular Tissue Engineering, LLC. This research work is independent and objective. The other authors have no conflicts of interest to disclose.

\begin{abstract}
Abbreviations
ACS: acute coronary syndrome, AMI: acute myocardial infarction, STEMI: ST elevation myocardial infarction, UA: unstable angina, CAD: coronary artery disease, MI: myocardial injury, CK-MB: creatine kinase isoenzyme MB, CCrP: Cyclocreatine Phosphate, ED: emergency department, ELISA: enzyme-linked immunosorbent assay, DEGs: different expressed genes, FTHL-17: Ferritin heavy chain like 17, ANAPC11: anaphase promoting complex subunit, CTB89H12.4: long coding RNA, min: minimum, max: maximum, GO: gene ontology, KEGG: Kyoto Encyclopedia of Genes and Genomes, LV: left ventricular, lncR: long non-coding RNA, miR: micro RNA, mRNA: messenger RNA, TNF- $\alpha$ : tumor necrosis factor-alpha, VECs: vascular endothelial cells, PCI: percutaneous coronary intervention, Isoproterenol: ISO.
\end{abstract}

\title{
References
}

1. Sanchis-Gomar, F.; Perez-Quilis, C.; Leischik, R.; Lucia, A. Epidemiology of coronary heart disease and acute coronary syndrome. Ann. Transl. Med. 2016, 4, 256. [CrossRef]

2. Stepinska, J.; Lettino, M.; Ahrens, I.; Bueno, H.; Garcia-Castrillo, L.; Khoury, A.; Lancellotti, P.; Mueller, C.; Muenzel, T.; Oleksiak, A.; et al. Diagnosis and risk stratification of chest pain patients in the emergency department: Focus on acute coronary syndromes. A position paper of the Acute Cardiovascular Care Association. Eur. Heart J. Acute Cardiovasc. Care 2020, 9, 76-89. [CrossRef]

3. Kossaify, A.; Bassil, E.; Kossaify, M. Stress Echocardiography: Concept and Criteria, Structure and Steps, Obstacles and Outcomes, Focused Update and Review. Cardiol. Res. 2020, 11, 89-96. [CrossRef] [PubMed]

4. Deepti, S.; Roy, A.; Patel, C.D.; Tandon, N.; Naik, N.; Singh, S.; Sharma, G.; Bahl, V.K. Assessment of asymptomatic ischemic heart disease using stress myocardial perfusion imaging in patients with type 2 diabetes mellitus. Indian Heart J. 2018, 70, S157-S160. [CrossRef]

5. Murthy, V.L.; Bateman, T.M.; Chen, W.; Malhotra, S.; Miller, E.J.; Ruddy, T.D.; Dilsizian, V. Impact of the ISCHEMIA Trial on Stress Nuclear Myocardial Perfusion Imaging. J. Nucl. Med. 2020, 61, 962-964. [CrossRef]

6. Garzillo, C.L.; Hueb, W.; Gersh, B.; Rezende, P.C.; Lima, E.G.; Favarato, D.; Ramires, J.A.F.; Filho, R.K. Association Between Stress Testing-Induced Myocardial Ischemia and Clinical Events in Patients With Multivessel Coronary Artery Disease. JAMA Intern. Med. 2019, 179, 1345. [CrossRef] [PubMed]

7. $\quad$ Chung, S.-Y.; Lee, K.-Y.; Chun, E.J.; Lee, W.-W.; Park, E.K.; Chang, H.-J.; Choi, S.I. Comparison of Stress Perfusion MRI and SPECT for Detection of Myocardial Ischemia in Patients With Angiographically Proven Three-Vessel Coronary Artery Disease. Am. J. Roentgenol. 2010, 195, 356-362. [CrossRef] [PubMed]

8. Heusch, G. Myocardial ischemia: Lack of coronary blood flow, myocardial oxygen supply-demand imbalance, or what? Am. J. Physiol. Circ. Physiol. 2019, 316, H1439-H1446. [CrossRef] [PubMed]

9. Heusch, G.; Gersh, B.J. The pathophysiology of acute myocardial infarction and strategies of protection beyond reperfusion: A continual challenge. Eur. Heart J. 2016, 38, 224-784. [CrossRef]

10. Reed, G.W.; Rossi, J.E.; Cannon, C.P. Acute myocardial infarction. Lancet 2017, 389, 197-210. [CrossRef]

11. Shah, A.S.V.; Ferry, A.V.; Mills, N.L. Cardiac Biomarkers and the Diagnosis of Myocardial Infarction in Women. Curr. Cardiol. Rep. 2017, 19, 1-10. [CrossRef]

12. Giannitsis, E.; Gopi, V. Biomarkers for infarct diagnosis and rapid rule-out/rule-in of acute myocardial infarction. Herz 2020, 45 , 1-9. [CrossRef] [PubMed] 
13. Aydin, S.; Ugur, K.; Aydin, S.; Sahin, I.; Yardim, M. Biomarkers in acute myocardial infarction: Current perspectives. Vasc. Health Risk Manag. 2019, 15, 1-10. [CrossRef] [PubMed]

14. Chen, Y.; Tao, Y.; Zhang, L.; Xu, W.; Zhou, X. Diagnostic and prognostic value of biomarkers in acute myocardial infarction. Postgrad. Med. J. 2019, 95, 210-216. [CrossRef] [PubMed]

15. Park, K.C.; Gaze, D.C.; Collinson, P.O.; Marber, M.S. Cardiac troponins: From myocardial infarction to chronic disease. Cardiovasc. Res. 2017, 113, 1708-1718. [CrossRef] [PubMed]

16. Elgebaly, S.A.; Poston, R.; Todd, R.; Helmy, T.; Almaghraby, A.M.; Elbayoumi, T.; Kreutzer, D.L.; Taegtmeyer, H.; Elgebaly, S. Cyclocreatine protects against ischemic injury and enhances cardiac recovery during early reperfusion. Expert Rev. Cardiovasc. Ther. 2019, 17, 683-697. [CrossRef] [PubMed]

17. Elgebaly, S.A.; Hashmi, F.H.; Houser, S.L.; Allam, M.E.; Doyle, K. Cardiac-derived neutrophil chemotactic factors: Detection in coronary sinus effluents of patients undergoing myocardial revascularization. J. Thorac. Cardiovasc. Surg. 1992, 103, 952-959. [CrossRef]

18. Elgebaly, S.A.; Elgebaly, S. Cardiac derived neutrophil chemotactic factors; preliminary biochemical characterization. J. Mol. Cell. Cardiol. 1989, 21, 585-593. [CrossRef]

19. Elgebaly, S.A.; Van Buren, C.; Todd, R.; Poston, R.; Rabie, M.A.; Mohamed, A.F.; Ahmed, L.A.; Sayed, N.S.E. Abstract 13311: Cyclocreatine Phosphate: A Novel Mechanism for Preventing Development of Heart Failure. Circulation 2020, 142 , A13311. [CrossRef]

20. Elgebaly, S.A. Nourexal: A Novel Anti-inflammatory/Antiapoptotic Therapy Against Reperfusion Injury. J. Clin. Exp. Cardiol. 2016, 7, 33 .

21. Elgebaly, S.; Christenson, R.; Kandil, H.; El-Khazragy, N.; Rashed, L.; Yacoub, B.; Eldeeb, H.; Ali, M.; Sharafieh, R.; Klueh, U.; et al. Nourin-Dependent miR-137 and miR-106b: Novel Early Inflammatory Diagnostic Biomarkers for Unstable Angina Patients. Biomolecules 2021, 11, 368. [CrossRef] [PubMed]

22. Elgebaly, S.A.; Christenson, R.H.; Kandil, H.; El-Khazragy, N.; Rashed, L.; Yacoub, B.; Sharafieh, R.; Klueh, U.; Kreutzer, D.L. Abstract 13051: Nourin-dependent Mirna-137: A Novel Early Diagnostic Biomarker for Unstable Angina Patients. Circulation 2020, 142, A13051. [CrossRef]

23. Elgebaly, S.A.; Christenson, R.H.; Kandil, H.; Elkhazragy, N.; Rashed, L.; Yacoub, B.; Sharafieh, R.; Klueh, U.; Kreutzer, D.L. Abstract 13103: Nourin-dependent Mirna-106b: A Novel Early Inflammatory Diagnostic Biomarker for Cardiac Injury. Circulation 2020, 142, A13103. [CrossRef]

24. Elgebaly, S.; Todd, R.; Kreutzer, D.; Christenson, R.; El-Khazragy, N.; Arafa, R.; Rabie, M.; Mohamed, A.; Ahmed, L.; El Sayed, N. Nourin-Associated miRNAs: Novel Inflammatory Monitoring Markers for Cyclocreatine Phosphate Therapy in Heart Failure. Int. J. Mol. Sci. 2021, 22, 3575. [CrossRef] [PubMed]

25. Elgebaly, S.; Christenson, R.; Schiffmann, E.; Yi, Q.; Kreutzer, D. Early identification of cardiac ischemia patients in the emergency department. Catheter. Cardiovasc. Interv. 2013, 81, S2-S3.

26. Zaghloul, A.; Iorgoveanu, C.; Balakumaran, K.; Balanescu, D.V.; Donisan, T. Limitations of Coronary Computed Tomography Angiography in Predicting Acute Coronary Syndrome in a Low to Intermediate-risk Patient with Chest Pain. Cureus 2018, 10, e2649. [CrossRef]

27. Rahsepar, A.A.; Arbab-Zadeh, A. Cardiac CT vs. Stress Testing in Patients with Suspected Coronary Artery Disease: Review and Expert Recommendations. Curr. Cardiovasc. Imaging Rep. 2015, 8, 29. [CrossRef]

28. Arbab-Zadeh, A. Stress Testing and Non-Invasive Coronary Angiography in Patients with Suspected Coronary Artery Disease: Time for a New Paradigm. Heart Int. 2012, 7, e2. [CrossRef]

29. Zhang, L.; Zhang, Y.; Zhao, Y.; Wang, Y.; Ding, H.; Xue, S.; Li, P. Circulating miRNAs as biomarkers for early diagnosis of coronary artery disease. Expert Opin. Ther. Pat. 2018, 28, 591-601. [CrossRef]

30. Adamson, P.D.; Hunter, A.; Madsen, D.M.; Shah, A.S.V.; McAllister, D.A.; Pawade, T.A.; Williams, M.C.; Berry, C.; Boon, N.A.; Flather, M.; et al. High-Sensitivity Cardiac Troponin I and the Diagnosis of Coronary Artery Disease in Patients With Suspected Angina Pectoris. Circ. Cardiovasc. Qual. Outcomes 2018, 11. [CrossRef]

31. Wongpraparut, N.; Piyophirapong, S.; Maneesai, A.; Sribhen, K.; Krittayaphong, R.; Bsc, R.P.; White, H.D. High-Sensitivity Cardiac Troponin T in Stable Patients Undergoing Pharmacological Stress Testing. Clin. Cardiol. 2015, 38, 293-299. [CrossRef] [PubMed]

32. Schulz, O.; Paul-Walter, C.; Lehmann, M.; Abraham, K.; Berghöfer, G.; Schimke, I.; Jaffe, A.S. Usefulness of Detectable Levels of Troponin, Below the 99th Percentile of the Normal Range, as a Clue to the Presence of Underlying Coronary Artery Disease. Am. J. Cardiol. 2007, 100, 764-769. [CrossRef] [PubMed]

33. Sabatine, M.S.; Morrow, D.A.; De Lemos, J.A.; Jarolim, P.; Braunwald, E. Detection of acute changes in circulating troponin in the setting of transient stress test-induced myocardial ischaemia using an ultrasensitive assay: Results from TIMI 35 . Eur. Heart J. 2008, 30, 162-169. [CrossRef] [PubMed]

34. Kurz, K.; Giannitsis, E.; Zehelein, J.; Katus, H.A. Highly Sensitive Cardiac Troponin T Values Remain Constant after Brief Exerciseor Pharmacologic-Induced Reversible Myocardial Ischemia. Clin. Chem. 2008, 54, 1234-1238. [CrossRef]

35. Samaha, E.; Avila, A.; Helwani, M.A.; Ben Abdallah, A.; Jaffe, A.S.; Scott, M.G.; Nagele, P. High-Sensitivity Cardiac Troponin After Cardiac Stress Test: A Systematic Review and Meta-Analysis. J. Am. Heart Assoc. 2019, 8, e008626. [CrossRef] 
36. Elbaz, M.; Faccini, J.; Laperche, C.; Grousset, E.; Roncalli, J.; Ruidavets, J.-B.; Vindis, C. Identification of a miRNA Based-Signature Associated with Acute Coronary Syndrome: Evidence from the FLORINF Study. J. Clin. Med. 2020, 9, 1674. [CrossRef]

37. Wang, L.; Jin, Y. Noncoding RNAs as Biomarkers for Acute Coronary Syndrome. BioMed Res. Int. 2020, 2020, 3298696. [CrossRef]

38. Kaur, A.; Mackin, S.T.; Schlosser, K.; Wong, F.L.; Elharram, M.; Delles, C.; Stewart, D.J.; Dayan, N.; Landry, T.; Pilote, L. Systematic review of microRNA biomarkers in acute coronary syndrome and stable coronary artery disease. Cardiovasc. Res. 2019, 116, 1113-1124. [CrossRef]

39. Zhang, P.; Liang, T.; Chen, Y.; Wang, X.; Wu, T.; Xie, Z.; Luo, J.; Yu, Y.; Yu, H. Circulating Exosomal miRNAs as Novel Biomarkers for Stable Coronary Artery Disease. BioMed Res. Int. 2020, 2020, 1-11. [CrossRef]

40. Kumar, D.; Narang, R.; Sreenivas, V.; Rastogi, V.; Bhatia, J.; Saluja, D.; Srivastava, K. Circulating miR-133b and miR-21: Potential non-invasive diagnostic biomarkers of coronary artery disease. Eur. Heart J. 2020, 41, 41. [CrossRef]

41. Melak, T.; Baynes, H.W. Circulating microRNAs as possible biomarkers for coronary artery disease: A narrative review. EJIFCC 2019, 30, 179-194.

42. Singh, S.; De Ronde, M.W.J.; Kok, M.G.M.; Beijk, M.A.; De Winter, R.J.; Van Der Wal, A.C.; Sondermeijer, B.M.; Meijers, J.C.M.; Creemers, E.E.; Pinto-Sietsma, S.-J. MiR-223-3p and miR-122-5p as circulating biomarkers for plaque instability. Open Heart 2020, 7, e001223. [CrossRef]

43. Zhao, T.; Qiu, Z.; Gao, Y. MiR-137-3p exacerbates the ischemia-reperfusion injured cardiomyocyte apoptosis by targeting KLF15. Naunyn-Schmiedeberg's Arch. Pharmacol. 2019, 393, 1013-1024. [CrossRef]

44. Liu, Z.; Yang, D.; Xie, P.; Ren, G.; Sun, G.; Zeng, X.; Sun, X. MiR-106b and MiR-15b Modulate Apoptosis and Angiogenesis in Myocardial Infarction. Cell. Physiol. Biochem. 2012, 29, 851-862. [CrossRef]

45. Cortigiani, L.; Picano, E.; Coletta, C.; Chiarella, F.; Mathias, W.; Gandolfo, N.; De Alcantara, M.; Mazzoni, V.; Gensini, G.F.; Landi, P. Safety, feasibility, and prognostic implications of pharmacologic stress echocardiography in 1482 patients evaluated in an ambulatory setting. Am. Heart J. 2001, 141, 621-629. [CrossRef]

46. Rozanski, A.; Gransar, H.; Hayes, S.W.; Min, J.; Friedman, J.D.; Thomson, L.E.; Berman, D.S. Temporal Trends in the Frequency of Inducible Myocardial Ischemia During Cardiac Stress Testing: 1991 to 2009. J. Am. Coll. Cardiol. 2013, 61, 1054-1065. [CrossRef]

47. Collet, J.P.; Thiele, H.; Barbato, E.; Barthélémy, O.; Bauersachs, J.; Bhatt, D.L.; Dendale, P.; Dorobantu, M.; Edvardsen, T.; Folliguet, T.; et al. 2020 ESC Guidelines for the management of acute coronary syndromes in patients presenting without persistent ST-segment elevation. Eur. Heart J. 2021, 42, 1289-1367. [CrossRef]

48. Thomas, G.S.; Voros, S.; McPherson, J.A.; Lansky, A.J.; Winn, M.E.; Bateman, T.M.; Elashoff, M.R.; Lieu, H.D.; Johnson, A.M.; Daniels, S.E.; et al. A Blood-Based Gene Expression Test for Obstructive Coronary Artery Disease Tested in Symptomatic Nondiabetic Patients Referred for Myocardial Perfusion Imaging the COMPASS Study. Circ. Cardiovasc. Genet. 2013, 6, 154-162. [CrossRef]

49. Bates, E.R.; Tamis-Holland, J.E.; Bittl, J.A.; O'Gara, P.T.; Levine, G.N. PCI Strategies in Patients With ST-Segment Elevation Myocardial Infarction and Multivessel Coronary Artery Disease. J. Am. Coll. Cardiol. 2016, 68, 1066-1081. [CrossRef] [PubMed]

50. Zhang, Z.; Jones, P.; Weintraub, W.S.; Mancini, G.J.; Sedlis, S.; Maron, D.J.; Teo, K.; Hartigan, P.; Kostuk, W.; Berman, D.; et al. Predicting the Benefits of Percutaneous Coronary Intervention on 1-Year Angina and Quality of Life in Stable Ischemic Heart Disease. Circ. Cardiovasc. Qual. Outcomes 2018, 11, e003971. [CrossRef] 